\title{
Síndrome de risco cognitivo motor em pessoas idosas de um serviço de saúde do Distrito Federal: estudo transversal
}

\author{
Motoric cognitive risk syndrome in older adults at a health service in the Distrito Federal: a cross- \\ sectional study
}

Tayla Gomes de Moura' ID Hudson Azevedo Pinheiro, $\mathbb{1 D}^{\mathrm{D}}$

\section{Resumo}

Objetivo: Verificar a prevalência da síndrome de risco cognitivo motor (SRCM) em pessoas idosas do Distrito Federal (DF), Brasil, que frequentaram os serviços de saúde de uma unidade de referência em Geriatria e Gerontologia, comparar os grupos de idosos com e sem a síndrome e investigar os possíveis fatores associados ao desenvolvimento dessa síndrome. Método: Trata-se de estudo observacional transversal analítico, desenvolvido com idosos (idade $\geq 60$ anos) com marcha independente e sem alterações cognitivas graves, que possuíam registro de dados sociodemográficos, avaliação cognitiva, capacidade funcional e velocidade de marcha em prontuários datados de 2017 a 2019. A análise dos dados foi expressa em média e desvio-padrão, frequência e percentual, e em odds ratios (OR) com intervalos de confiança de 95\%. As comparações entre os grupos com e sem a SRCM foram feitas por meio dos testes: qui-quadrado, U de Mann-Whitney e t de Student. Resultados: Não houve diferenças significativas na comparação das variáveis entre os grupos. A prevalência da SRCM na população estudada foi de $24 \%$. Nenhum dos fatores analisados demonstrou associação com a presença da síndrome. Conclusão: A prevalência da SRCM na amostra foi de $24 \%$ e mostrou-se mais alta na população do DF quando comparada as populações estudadas em outros países. Não houve diferenças entre os grupos de idosos com e sem a síndrome, e os fatores associados não foram encontrados. O rastreio da síndrome é de extrema relevância, pois a partir desses achados pode-se desenvolver mecanismos para a prevenção de demência em idosos.

\footnotetext{
Fundação de Ensino e Pesquisa em Ciências da Saúde (FEPECS), Secretaria de Saúde do Distrito Federal, Programa de Residência Multiprofissional em Saúde do Adulto e do Idoso. Brasília, DF, Brasil.

2 Centro Universitário do Distrito Federal (UDF/Cruzeiro do Sul), Curso de Fisioterapia. Brasília, DF, Brasil.
}

Palavras-chave: Saúde do Idoso. Síndrome. Disfunção Cognitiva. Velocidade de caminhada. Demência.

Os autores declaram não haver conflito na concepção deste trabalho.

Não houve financiamento para a execução deste trabalho. 


\section{Abstract}

Objective: To verify the prevalence of the motoric cognitive risk syndrome (MCR) in older adults from the Distrito Federal (DF), Brazil, who attended the health services of a reference unit in Geriatrics and Gerontology, compare groups of older adults with and without the syndrome and investigate the possible associated factors for the development of this syndrome. Method: This is an observational cross-sectional analytical study, developed with older adults (age $\geq 60$ years) with independent gait and without severe cognitive dysfunctions, who had a record of sociodemographic data, cognitive assessment, functional capacity and gait speed in medical records dated 2017 to 2019. Data analysis was expressed as mean and standard deviation, frequency and percentage, and odds ratios (OR) with 95\% confidence intervals. Comparisons between groups with and without MCR were made using the chi-square, U Mann Whitney and t-student tests. Results: There were no significant differences in the comparison of variables between groups. The prevalence of MCR in the studied population was $24 \%$. None of the factors analyzed showed an association with the presence of the syndrome. Conclusion: The prevalence of MCR in the sample was $24 \%$ and was shown to be higher in the population of the DF when compared to the populations studied in other countries. There were no differences between the groups of older adults with and without the syndrome, and the associated factors were not found. Screening for the syndrome is hugely relevant, as, from these findings, mechanisms can be developed to prevent dementia in old people.

\section{INTRODUÇÃO}

O processo de envelhecimento repercute em diversas alterações fisiológicas em todos os níveis nos sistemas do organismo, sendo a principal característica desse processo a diminuição da reserva fisiológica, com consequente declínio na capacidade funcional do idoso ${ }^{1}$. A vulnerabilidade aos danos, decorrente das falhas que surgem nas moléculas, células e tecidos do corpo ao longo do processo de envelhecimento, pode favorecer a aquisição de doenças relacionadas à idade, sendo que estas tem potencial para gerar incapacidade e fragilidade ${ }^{2}$.

Dentro desse processo de envelhecimento, merece destaque o envelhecimento cerebral, que é caracterizado pelo número de mudanças neurobiológicas, anatômicas, metabólicas, neuroquímicas e nos circuitos neuronais, repercutindo diretamente sobre as funções sensório motora e cognitiva dos idosos ${ }^{3,4}$, sendo considerado um importante marcador em relação à capacidade funcional desse grupo 5 .

Outro importante marcador da capacidade funcional de idosos diz respeito à velocidade da marcha, que atua como um marcador da função motora e das funções cognitivas. Em idosos saudáveis, a velocidade da marcha apresenta relações significativas com a pontuação em testes de avaliação cognitiva ${ }^{6}$, sendo que a redução da velocidade da marcha tem sido demonstrada como fator preditivo do declínio cognitivo nessa população ${ }^{6-9}$.

Neste contexto, a síndrome de risco cognitivo motor (SRCM) surge como uma alternativa para a detecção precoce do risco de desenvolvimento de demência e disfunções/incapacidades, sendo definida como a presença de queixas cognitivas e lentidão da marcha em idosos que não possuam diagnóstico de demência ou incapacidade de mobilidade ${ }^{10,11}$. O rastreio dessa síndrome é de extrema relevância, pois sua presença tem sido associada a fatores de risco frequentes na população brasileira, como doenças cardíacas, doenças crônicas e deficit visual ${ }^{12-15}$, além de ter se mostrado como preditiva do desenvolvimento de demências, risco de quedas, disfunções/incapacidades e mortalidade em idosos ${ }^{16,17}$.

Além disso, o Brasil encontra-se como o segundo país com maior prevalência de demência a nível global $^{18}$, o que favorece a hipótese de que a SRCM pode ser mais prevalente na nossa população de
Keywords: Health of the
Eldely. Syndrome. Cognitive Dysfunction. Walking Speed. Dementia. 
idosos. Assim, o presente estudo teve por objetivo verificar a prevalência da SRCM em pessoas idosas do Distrito Federal (DF) que frequentaram os serviços de saúde de uma unidade de referência em Geriatria e Gerontologia, comparar os grupos de idosos com e sem a síndrome e investigar os possíveis fatores associados ao desenvolvimento dessa síndrome na população descrita.

\section{MÉTODO}

O presente estudo foi desenvolvido como uma pesquisa do tipo observacional, de caráter transversal e analítico utilizando dados secundários provenientes de prontuários médicos.

O estudo foi realizado por meio de uma amostra de conveniência, sendo que os dados foram coletados dos prontuários de pacientes que foram encaminhados pelas Unidades Básicas de
Saúde (UBS) da região e admitidos no serviço de acolhimento de idosos de uma unidade de referência em Geriatria e Gerontologia (nível secundário), entre os anos de 2017 a 2019.

A Figura 1 apresenta o fluxograma dos usuários que foram acolhidos na unidade de referência em Geriatria e Gerontologia e sua distribuição de acordo com os critérios de inclusão e exclusão.

O acolhimento era realizado por uma equipe multidisciplinar composta por um enfermeiro, um fisioterapeuta, um nutricionista e um assistente social ou psicólogo. Essa equipe era responsável por realizar uma anamnese baseada nos critérios da avaliação geriátrica ampla (AGA) $)^{19}$ e por realizar testes de rastreio da função cognitivaa ${ }^{20}$, capacidade funcional ${ }^{21-25}$ e de vulnerabilidade social/psicológica, sendo que após a realização dessa avaliação, a equipe definia se o usuário seria admitido nesta unidade ou se seria feita a contrarreferência para a UBS de origem.

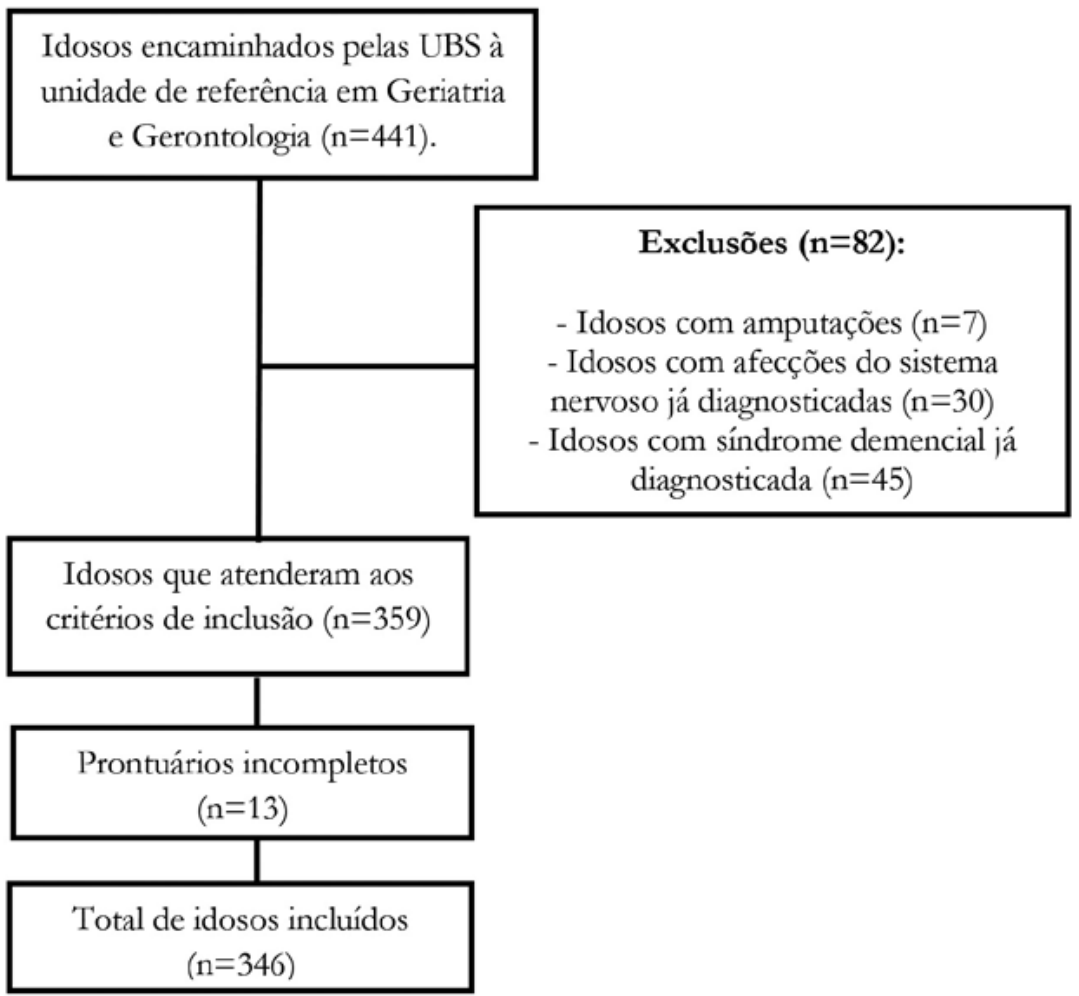

Figura 1. Fluxograma de captação da amostra. Taguatinga, DF, 2017 a 2019. 
Os resultados dessas avaliações realizadas durante o acolhimento eram registrados em planilhas, por meio de um programa de computador, e nos prontuários dos idosos, sendo que a coleta dos dados utilizados neste estudo (e descritos a posteriori) foi feita por meio desses registros do acolhimento.

Foram incluídos neste estudo os dados dos prontuários de pessoas idosas com idade igual ou superior a 60 anos, capazes de deambular por meio de marcha independente e que realizaram os testes de avaliação da velocidade da marcha e do desempenho cognitivo, assim como de independência para a realização das atividades de vida diária ${ }^{10}$.

Adotou-se como critérios de exclusão: idosos com disfunção motora grave já instalada, com diagnóstico prévio de síndrome demencial, ainda que a doença estivesse em seu estágio inicial ${ }^{10,11}$, além de outras afecções do sistema nervoso (ex. sequelas de Acidente Vascular Encefálico, Doença de Parkinson, entre outras) e amputações. Também foram excluídos do estudo os dados de prontuários que não estavam totalmente preenchidos e que as informações perdidas não puderam ser resgatadas.

Em relação aos critérios diagnósticos da SRCM, para que sua presença fosse reconhecida, o idoso precisava apresentar quatro características: I) queixas cognitivas avaliadas em testes neuropsicológicos, II) lentidão da marcha, caracterizada como velocidade de marcha um desvio-padrão ou mais abaixo do previsto, de acordo com a idade e o sexo, III) capacidade preservada para a realização das suas atividades de vida diária (AVD), e IV) ausência do diagnóstico de demência ${ }^{10}$.

Nesse estudo, para detectar a presença da SRCM, a função cognitiva foi mensurada por meio do Miniexame do Estado Mental (MEEM), que avalia a presença de alterações cognitivas. Os pontos de corte adotados para a existência de alterações no MEEM foram: para idosos analfabetos, escore no teste menor do que 18 pontos; para idosos com até quatro anos de estudo, escore no teste menor do que 24 pontos; para idosos com até oito anos de estudo, escore no teste menor do que 26 pontos; e para idosos com escolaridade maior ou igual a nove anos de estudo, escore no teste menor do que 27 pontos de um total de 30 pontos, sendo avaliados os domínios cognitivos de orientação temporal, orientação espacial, memória imediata, atenção e cálculo, e evocação e linguagem ${ }^{20}$.

A detecção de lentidão na marcha foi feita por meio do teste de velocidade habitual da marcha (VHM). Em um corredor de aproximadamente 10 metros de comprimento, os participantes foram orientados a caminhar em uma velocidade confortável a distância referida, sendo excluídos os primeiros três e os últimos quatro metros de deslocamento. O tempo para a execução do teste foi cronometrado e o valor da VHM foi determinado pela divisão da distância percorrida pelo tempo obtido no cronômetro, sendo adotado como pontuação de corte o valor de 0.8 metros/segundo 24,26 .

A avaliação da habilidade dos idosos para a realização das suas AVD foi feita por meio de dois instrumentos: o índice de Katz, para mensurar o grau de independência para as atividades básicas de vida diária (ABVD) ${ }^{21}$, e o questionário de Lawton \& Brody, para avaliar o grau de independência para as atividades instrumentais de vida diária (AIVD) ${ }^{22}$.

Os participantes foram classificados como independentes para as ABVD quando apresentaram escore no teste igual a 0 , necessitam de assistência para as ABVD quando apresentaram escores no teste variando entre 1 e 5 pontos, e totalmente dependentes para as ABVD quando apresentaram escore no teste igual a 6 pontos $^{21,23}$. Já para as AIVD, os participantes foram considerados independentes quando apresentaram escore no teste igual a 27 pontos, necessitam de assistência quando apresentaram escores no teste variando entre 18 e 26 pontos, e totalmente dependentes quando apresentaram escores no teste variando entre 9 e 17 pontos $^{25}$.

Uma vez por semana, os dois pesquisadores envolvidos no estudo acessaram o sistema de registro de dados dos pacientes da unidade de referência em Geriatria e Gerontologia (DF) e recolheram os dados necessários para a pesquisa, sendo que essa coleta ocorreu entre os meses de março e maio de 2020.

Os dados foram analisados descritivamente utilizando medidas de tendência central (média e mediana) e de variabilidade (desvio padrão e 
amplitude interquartil [percentis $25 \%$ e $75 \%$ ]) para dados contínuos e medidas de frequência absoluta e relativa para dados categóricos. A distribuição dos dados contínuos foi analisada por meio do teste Kolmogorov-Smirnov. A comparação dos dados categóricos entre os grupos de idosos com e sem a SRCM foi analisada utilizando o teste qui-quadrado, e dos dados numéricos contínuos utilizando o teste U de Mann-Whitney (dados não paramétricos) e teste t de Student independente (dados paramétricos). Os valores dos índices de w foram calculados como medidas dos tamanhos de efeito entre os grupos, sendo considerado efeito pequeno $(\mathrm{d}=0,20-0,49$ e $\mathrm{w}=010-0,29)$, efeito médio $(\mathrm{d}=0,50-0,79 \mathrm{e} \mathrm{w}=0,30$ $0,49)$ e efeito grande ( $d \geq 0,80$ e w $\geq 0,50)$. Foi utilizada a regressão logística univariada para determinar associações entre as características investigadas com a identificação da SRCM. As odds ratios (OR) com intervalo de confiança de $95 \%$ foram calculadas para cada variável independente. O nível de significância de $5 \%$ foi considerado.

Respeitando os aspectos éticos abordados nas Resoluções no 466/2012 e n n 510/2016, esta pesquisa foi submetida e aprovada pelo Comitê de Ética em Pesquisa (CEP) da Secretaria de Estado de Saúde do Distrito Federal (SES/DF) (Parecer 4.198.150/2020). A coleta de dados foi realizada com base em pesquisa de prontuário, havendo dispensa da assinatura do Termo de Consentimento Livre e Esclarecido (TCLE) pelos participantes.

\section{RESULTADOS}

As características clínicas e sociodemográficas dos idosos que compuseram a amostra deste estudo estão apresentadas na Tabela 1. As análises indicaram que houve maior predomínio de idosos do sexo feminino na amostra (71,4\%). Além disso, a maioria $(76,0 \%)$ dos idosos apresentou baixa escolaridade (analfabetismo ou ensino fundamental), polifarmácia $(68,5 \%)$ e independência para a realização das atividades básicas $(75,7 \%)$ e instrumentais de vida diária $(57,5 \%)$.

Neste estudo, 24\% dos idosos investigados apresentaram a SRCM. Os critérios identificados e analisados em cada grupo estão apresentados na Tabela 2. Entre o grupo sem a SRCM, o critério para a síndrome mais prevalente foi a independência para as ABVD, seguido do deficit cognitivo e da lentidão da marcha. Todos os critérios apresentaram diferença significativa $(p<0,001)$ na comparação entre os grupos de idosos com e sem a SRCM.

Nenhum dos fatores examinados demonstrou diferença entre os grupos de estudo. A comparação desses fatores entre os grupos está apresentada na Tabela 3.

$\mathrm{Na}$ análise de regressão logística univariada, nenhum dos fatores analisados mostrou-se como fator associado à presença da SRCM. Esse resultado está apresentado na Tabela 4. 
Tabela 1. Características clínicas e sociodemográficas da amostra (N=346). Taguatinga, DF, 2017 a 2019.

\begin{tabular}{|c|c|}
\hline Variável & Amostra Geral \\
\hline \multicolumn{2}{|l|}{ Sexo, $\%(n)$} \\
\hline Feminino & $71,40(247)$ \\
\hline Masculino & $28,60(99)$ \\
\hline \multicolumn{2}{|l|}{ Idade } \\
\hline média (DP) & $77,71( \pm 8,10)$ \\
\hline mediana [Percentil 25\%; 75\%] & $78,00[72 ; 84]$ \\
\hline \multicolumn{2}{|l|}{ Escolaridade, \% (n) } \\
\hline Analfabeto & $28,60(99)$ \\
\hline Ensino Fundamental & $47,40(164)$ \\
\hline Ensino Médio & $19,70(68)$ \\
\hline Ensino Superior & $4,30(15)$ \\
\hline \multicolumn{2}{|l|}{ Diagnósticos, \% (n) } \\
\hline Hipertensão arterial sistêmica (sim) & $74,30(257)$ \\
\hline Diabetes Mellitus (sim) & $34,40(119)$ \\
\hline Alterações musculoesqueléticas (sim) & $42,80(148)$ \\
\hline Depressão (sim) & $23,80(82)$ \\
\hline \multicolumn{2}{|l|}{ Quantidade de medicamentos } \\
\hline média (DP) & $5,55( \pm 3,35)$ \\
\hline mediana [Percentil 25\%; 75\%] & $5,00[3 ; 8]$ \\
\hline Polifarmácia (sim), \% (n) & $68,50(237)$ \\
\hline \multicolumn{2}{|l|}{ IMC } \\
\hline média (DP) & $26,11( \pm 4,88)$ \\
\hline mediana [Percentil 25\%; 75\%] & $25,63[22,87 ; 28,52]$ \\
\hline \multicolumn{2}{|l|}{ Estado Nutricional, \% (n) } \\
\hline Baixo Peso (IMC<22 Kg/m²) & $18,20(63)$ \\
\hline Eutrofia $\left(\mathrm{IMC}=22\right.$ a $\left.27 \mathrm{Kg} / \mathrm{m}^{2}\right)$ & $43,60(151)$ \\
\hline Excesso de Peso $\left(\mathrm{IMC}>27 \mathrm{Kg} / \mathrm{m}^{2}\right)$ & $38,20(132)$ \\
\hline Exposição ao cigarro (sim), \% (n) & $31,50(109)$ \\
\hline Etilismo (sim), \% (n) & $14,70(51)$ \\
\hline Prática de exercício físico (sim), \% (n) & $23,40(81)$ \\
\hline Histórico de queda (sim), \% (n) & $34,10(118)$ \\
\hline \multicolumn{2}{|l|}{ Atividades básicas de vida diária, \% (n) } \\
\hline Independente & $75,70(262)$ \\
\hline Assistência & $15,90(55)$ \\
\hline Dependente & $8,40(29)$ \\
\hline \multicolumn{2}{|c|}{ Atividades instrumentais de vida diária, \% (n) } \\
\hline Independente & $57,50(199)$ \\
\hline Assistência & $24,90(86)$ \\
\hline Dependente & $17,60(61)$ \\
\hline
\end{tabular}

DP: desvio-padrão; IMC: índice de massa corporal. 
Tabela 2. Critérios diagnósticos para a presença da síndrome de risco cognitivo motor investigados ( $\mathrm{N}=346)$. Taguatinga, DF, 2017 a 2019.

\begin{tabular}{|c|c|c|c|}
\hline Variável & Amostra Geral & $\begin{array}{l}\text { Idosos sem SRCM } \\
(\mathrm{n}=263)\end{array}$ & $\begin{array}{l}\text { Idosos com SRCM } \\
(\mathrm{n}=83)\end{array}$ \\
\hline \multicolumn{4}{|l|}{ SRCM, \% (n) } \\
\hline Positivo & $24,00(83)$ & - & - \\
\hline Negativo & $76,00(263)$ & & \\
\hline \multicolumn{4}{|l|}{ Atividades básicas de vida diária, \% (n) } \\
\hline Independente & $75,70(262)$ & $68,10(179)$ & $100,00(83)$ \\
\hline Assistência & $15,90(55)$ & $20,90(55)$ & $0,00(0)$ \\
\hline Dependente & $8,40(29)$ & $11,00(29)$ & $0,00(0)$ \\
\hline Critério ABVD (sim-independente),\%(n) & $75,70(262)$ & $68,10(179)$ & $100,00(83)$ \\
\hline \multicolumn{4}{|l|}{ MEEM (escore) } \\
\hline média (DP) & $19,29( \pm 6,57)$ & $20,96(6,13)$ & $13,98(4,89)$ \\
\hline mediana [Percentil 25\%; 75\%] & $20,00[15 ; 24]$ & $22,00[18 ; 26]$ & $15,00[10 ; 17]$ \\
\hline Critério cognitivo (sim-deficit cognitivo),\%(n) & $64,70(224)$ & $53,60(141)$ & $100,00(83)$ \\
\hline \multicolumn{4}{|l|}{ Velocidade habitual de marcha $(\mathrm{m} / \mathrm{s})$} \\
\hline média (DP) & $0,83( \pm 0,30)$ & $0,90(0,30)$ & $0,61(0,14)$ \\
\hline mediana [Percentil 25\%; 75\%] & $0,80[0,60 ; 1,00]$ & $0,90[0,75 ; 1,00]$ & $0,60[0,50 ; 0,75]$ \\
\hline Critério marcha $(\operatorname{sim}-\mathrm{VHM}<0,8 \mathrm{~m} / \mathrm{s}), \%(\mathrm{n})$ & $47,70(165)$ & $31,20(82)$ & $100,00(83)$ \\
\hline
\end{tabular}

SRCM: Síndrome de risco cognitivo motor; MEEM: miniexame do estado mental; $p<0,001$.

Tabela 3. Comparação das variáveis em estudo entre os idosos com e sem a síndrome de risco cognitivo motor (N=346). Taguatinga, DF, 2017 a 2019.

\begin{tabular}{|c|c|c|c|c|}
\hline Variável & $\begin{array}{l}\text { Idosos sem } \\
\text { SRCM }(n=263)\end{array}$ & $\begin{array}{l}\text { Idosos com } \\
\text { SRCM }(n=83)\end{array}$ & $p$-valor & $\begin{array}{l}\text { Tamanho de } \\
\text { efeito (power) }\end{array}$ \\
\hline \multicolumn{5}{|l|}{ Sexo***, \% (n) } \\
\hline Feminino & $70,30(185)$ & $74,70(62)$ & 0,48 & $0,04(41 \%)$ \\
\hline Masculino & $29,70(78)$ & $25,30(21)$ & & \\
\hline \multicolumn{5}{|l|}{ Idade* } \\
\hline média (DP) & $77,49( \pm 8,10)$ & $78,42(8,12)$ & 0,36 & $0,11(15 \%)$ \\
\hline mediana [Percentil 25\%; 75\%] & $77,00[72 ; 83]$ & $78,00[72 ; 85]$ & & \\
\hline \multicolumn{5}{|l|}{ Escolaridade ${ }^{* * *}, \%$ (n) } \\
\hline Analfabeto & $27,40(72)$ & $32,50(27)$ & 0,14 & $0,12(41 \%)$ \\
\hline Ensino Fundamental & $45,60(120)$ & $53,00(44)$ & & \\
\hline Ensino Médio & $22,10(58)$ & $12,00(10)$ & & \\
\hline Ensino Superior & $4,90(13)$ & $2,40(2)$ & & \\
\hline \multicolumn{5}{|l|}{ Diagnósticos***, \% (n) } \\
\hline HAS (sim) & $75,30(198)$ & $71,10(59)$ & 0,47 & $0,04(41 \%)$ \\
\hline $\mathrm{DM}(\operatorname{sim})$ & $36,90(97)$ & $26,50(22)$ & 0,08 & $0,09(41 \%)$ \\
\hline Alterações musculoesqueléticas (sim) & $44,10(116)$ & $38,60(32)$ & 0,44 & $0,04(41 \%)$ \\
\hline Depressão (sim) & $24,00(63)$ & $22,90(19)$ & 0,88 & $0,01(41 \%)$ \\
\hline
\end{tabular}


Continuação da Tabela 3

\begin{tabular}{|c|c|c|c|c|}
\hline Variável & $\begin{array}{l}\text { Idosos sem } \\
\text { SRCM }(n=263)\end{array}$ & $\begin{array}{l}\text { Idosos com } \\
\text { SRCM }(n=83)\end{array}$ & $p$-valor & $\begin{array}{l}\text { Tamanho de } \\
\text { efeito (power) }\end{array}$ \\
\hline \multicolumn{5}{|l|}{ Quantidade de medicamentos** } \\
\hline média (DP) & $5,38( \pm 3,17)$ & $6,07(3,83)$ & 0,20 & $0,20(34 \%)$ \\
\hline mediana [Percentil 25\%; 75\%] & $5,00[3 ; 7]$ & $5,00[3 ; 9]$ & & \\
\hline Polifarmácia (sim) ${ }^{\text {c }}, \%$ (n) & $67,30(177)$ & $72,30(60)$ & 0,41 & $0,04(41 \%)$ \\
\hline \multicolumn{5}{|l|}{$\mathrm{IMC}^{* *}$} \\
\hline média (DP) & $25,99( \pm 4,86)$ & $26,51(4,92)$ & 0,48 & $0,11(13 \%)$ \\
\hline mediana [Percentil 25\%; 75\%] & $25,64[22,77 ; 28,30]$ & $25,58[23,07 ; 29,73]$ & & \\
\hline \multicolumn{5}{|l|}{ Estado Nutricional ${ }^{* * *}, \%$ (n) } \\
\hline Baixo Peso $\left(\mathrm{IMC}<22 \mathrm{Kg} / \mathrm{m}^{2}\right)$ & $19,40(51)$ & $14,50(12)$ & 0,57 & $0,06(41 \%)$ \\
\hline Eutrofia $\left(\mathrm{IMC}=22\right.$ a $\left.27 \mathrm{Kg} / \mathrm{m}^{2}\right)$ & $43,30(114)$ & $44,60(37)$ & & \\
\hline Excesso de Peso (IMC>27 Kg/m²) & $37,30(98)$ & $41,00(34)$ & & \\
\hline Exposição ao cigarro (sim)***, \% (n) & $31,60(83)$ & $31,30(26)$ & 0,96 & $0,002(41 \%)$ \\
\hline Prática de exercício físico (sim)***, \% (n) & $25,10(66)$ & $18,10(15)$ & 0,23 & $0,07(41 \%)$ \\
\hline Histórico de queda (sim)***, \% (n) & $34,60(91)$ & $32,50(27)$ & 0,79 & $0,02(41 \%)$ \\
\hline \multicolumn{5}{|l|}{ AIVD***, $\%(\mathrm{n})$} \\
\hline Independente & $57,40(151)$ & $57,80(48)$ & 0,20 & $0,09(41 \%)$ \\
\hline Assistência & $23,20(61)$ & $30,10(25)$ & & \\
\hline Dependente & $19,40(51)$ & $12,00(10)$ & & \\
\hline
\end{tabular}

SRCM: síndrome de risco cognitivo motor; HAS: hipertensão arterial sistêmica; DM: diabetes mellitus; IMC: índice de massa corporal; AIVD: atividades instrumentais de vida diária; "Variáveis com distribuição normal (teste Kolmogorov-Smirnov); Comparações intergrupo por meio do teste t de Student independente; ${ }^{* *}$ Variáveis com distribuição não-normal (teste Kolmogorov-Smirnov); Comparações intergrupo por meio do teste Mann-Whitney U; ${ }^{* * *}$ Variáveis categóricas; Comparações por meio do teste qui-quadrado.

Tabela 4. Análise de regressão logística univariada para identificar os fatores associados à síndrome de risco cognitivo motor na amostra (N=346). Taguatinga, DF, 2017 a 2019.

\begin{tabular}{lll}
\hline Variáveis & $\begin{array}{l}\text { Ocorrência da SRCM } \\
\text { OR [IC 95\%] }\end{array}$ & $p$-valor \\
\hline Sexo & $1,245[0,710 ; 2,182]$ & 0,444 \\
\hline Idade & $1,014[0,984 ; 1,046]$ & 0,359 \\
\hline DM & $0,617[0,357 ; 1,068]$ & 0,084 \\
\hline Depressão & $0,938[0,522 ; 1,684]$ & 0,830 \\
\hline HAS & $1,239[0,714 ; 2,150]$ & 0,446 \\
\hline Alterações musculoesqueléticas & $0,795[0,480 ; 1,317]$ & 0,373 \\
\hline Medicamentos & $1,062[0,988 ; 1,142]$ & 0,103 \\
\hline Polifarmácia & $0,789[0,457 ; 1,361]$ & 0,394 \\
\hline IMC & $1,021[0,972 ; 1,073]$ & 0,402 \\
\hline Atividade física & $0,658[0,353 ; 1,230]$ & 0,190 \\
\hline
\end{tabular}

SRCM: síndrome de risco cognitivo motor; OR = Odds Ratio; IC = Intervalo de Confiança; DM: diabetes mellitus; HAS: hipertensão arterial sistêmica; IMC: índice de massa corporal. 


\section{DISCUSSÃO}

Este estudo se propôs a avaliar a prevalência, comparar os grupos de idosos com e sem a SRCM e investigar os fatores associados a sua ocorrência, que tem se mostrado como preditiva de demência em idosos de outros países ${ }^{10,11,14,16,17,27}$.

A nossa hipótese consistia em uma prevalência maior da síndrome nos idosos brasileiros, quando comparados aos idosos do exterior, devido as condições socioeconômicas da população do país, especificamente do Distrito Federal. Os achados do presente estudo corroboram essa hipótese, sendo que na amostra dessa pesquisa encontrou-se uma prevalência de $24 \%$ de idosos com a SRCM, enquanto que em estudos anteriores, realizados em outros países, a prevalência da síndrome variou entre 2,65 a $12,8 \%$ da amostra ${ }^{13,14,28,29}$.

Entretanto, ressalta-se que essa maior taxa de prevalência da SRCM nos idosos deste estudo pode estar relacionada com a seleção de uma amostra por conveniência, utilizando dados de prontuários de idosos que frequentavam serviços de saúde, o que pode indicar uma amostra mais frágil e vulnerável, uma vez que esse grupo apresenta doenças que exigem maior complexidade de assistência à saúde, tendo sido encaminhados para um serviço de referência. Ademais, esse perfil de idosos (idosos que necessitam de serviços de saúde) se caracteriza por apresentar menor renda, baixa escolaridade, não praticar atividade física regularmente e apresentar risco elevado para a ocorrência de doenças cardiovasculares e metabólicas, por exemplo ${ }^{30}$, o que pode favorecer ainda mais a incapacidade funcional e outros agravos à saúde.

Além disso, em relação aos critérios diagnósticos da SRCM, no presente estudo, os idosos que apresentaram a síndrome tiveram pior desempenho no teste cognitivo e eram mais lentos para a marcha do que os idosos que não tinham a SRCM. Para explicar a diferença no desempenho cognitivo entre os idosos com e sem a síndrome, Maguire et al. ${ }^{14}$ avaliaram alguns domínios da função cognitiva e constataram que os idosos com a SRCM tinham pior desempenho nos testes de memória, cognição global e atenção sustentada que os idosos sem a síndrome. Já em relação as diferenças na velocidade da marcha de idosos com e sem a SRCM, Ayers e Verghese ${ }^{31}$ analisaram os parâmetros da marcha e detectaram que os idosos com a síndrome apresentaram menor comprimento da passada e menor tempo da fase de balanço quando comparados aos idosos sem a SRCM.

Na comparação entre os grupos de idosos com e sem a SRCM, deste estudo, não houve diferenças significativas entre os grupos para as variáveis baixa escolaridade, presença de doenças crônicas, polifarmácia, excesso de peso e inatividade física, o que diverge dos achados de pesquisas anteriores que demonstraram diferenças significativas para a presença dessas variáveis entre os grupos ${ }^{13,32}$.

Em acréscimo, estudos anteriores que analisaram os fatores associados à presença da SRCM encontraram que a prática de atividade física ${ }^{12,33}$, o excesso de peso $^{12,14,28,33}$, a depressão ${ }^{12,14,33}$, as doenças cardiovasculares (como a hipertensão arterial sistêmica) $)^{34}$, as alterações musculoesqueléticas (osteoporose) e a polifarmácia ${ }^{33}$, influenciam na ocorrência da síndrome, o que diverge dos achados do presente estudo, que não demonstrou relação desses mesmos fatores investigados com a presença da SRCM. Tal resultado pode ser explicado pelo tamanho da amostra deste estudo, que não foi suficiente para gerar um tamanho de efeito (poder) adequado $-80 \%$ - para nenhuma das variáveis analisadas, e pela seleção dos participantes por meio de uma amostra de conveniência proveniente de um serviço de saúde de média complexidade, sendo estas limitações da pesquisa.

Apesar dessas limitações, este foi um estudo pioneiro na investigação da SRCM na população do Brasil, especificamente do Distrito Federal, sendo uma pesquisa relevante porque essa síndrome tem sido relacionada não somente com a evolução para o quadro de demência, como também para outros agravos à saúde, como disfunções cerebrais e musculoesqueléticas - que repercutem em alterações cognitivas e motoras que contribuem para a fragilidade no idoso ${ }^{35}$; aumento do risco de queda e aumento da taxa de mortalidade ${ }^{16}$. 


\section{CONCLUSÃO}

Esta pesquisa verificou a prevalência, comparou dois grupos de idosos e analisou os possíveis fatores associados à síndrome de risco cognitivo motor, uma síndrome geriátrica preditiva de demência. $\mathrm{Na}$ amostra, selecionada por conveniência e de forma não probabilística, e proveniente de um serviço de saúde, a taxa de prevalência dessa síndrome mostrou-se mais alta do que as taxas registradas em outros estudos, mas não houve diferenças entre os grupos de idosos com e sem a síndrome para as variáveis em estudo, com exceção das variáveis diagnósticas, e os fatores associados a essa maior prevalência não foram encontrados.
Entretanto, esses achados são de extrema relevância para a prática clínica, pois ao detectar a prevalência da síndrome, pode-se desenvolver mecanismos para a prevenção e controle do desenvolvimento de demência em pessoas idosas, uma vez que o quadro demencial repercute na capacidade funcional e na qualidade de vida desse grupo. Outras pesquisas com um maior número de participantes são necessárias para definir a taxa de prevalência da síndrome em caráter nacional e para permitir a verificação dos fatores associados ao desenvolvimento da síndrome de risco cognitivo motor.

Editado por: Maria Helena Rodrigues Galvão

\section{REFERÊNCIAS}

1. Organização Mundial da Saúde. Resumo: Relatório Mundial de Envelhecimento e Saúde [Internet]. Estados Unidos: OMS; 2015 [acesso em 06 out. 2019]. Disponível em: https://apps.who.int/ iris/bitstream/handle/10665/186468/WHO_ FWC_ALC_15.01_por.pdf;jsessionid=9DB2B B89255CBBACF7B127016EFBEF79? sequence $=6$

2. Kirkwood TBL. A systematic look at an old problem. Nature. 2008;451(7):644-7.

3. Li S, Dinse H. Aging of the brain, sensorimotor, and cognitive processes. Neurosci Biobehav Rev. 2002;26(7):729-32.

4. Sekhon H, Allali G, Launay CP, Barden J, Szturm $\mathrm{T}$, Liu-Ambrose T, et al. Motoric cognitive risk syndrome, incident cognitive impairment and morphological brain abnormalities: systematic review and meta-analysis. Maturitas. 2019;123:4554. Disponível em: https://doi.org/10.1016/j. maturitas.2019.02.006

5. Davis JC, Nagamatsu LS, Hsu CL, Beattie BL, Liuambrose T. Self-efficacy is independently associated with brain volume in older women. Age Ageing. 2012;41(4):495-501.

6. Fitzpatrick AL, Buchanan CK, Nahin RL, Dekosky ST, Atkinson HH, Carlson MC, et al. Associations of Gait Speed and Other Measures of Physical Function With Cognition in a Healthy Cohort of Elderly Persons. J Gerontol Med Sci. 2007;62(11):1244-51.

7. Bruce-keller AJ, Brouillette RM, Tudor-locke C, Foil HC, Gahan WP, Nye DM, et al. Relationship Between Cognitive Domains, Physical Performance, and Gait in Elderly and Demented Subjects. J Alzheimer's Dis. 2012;30(4):899-908.
8. Mielke MM, Roberts RO, Savica R, Cha R, Drubach DI, Christianson T, et al. Assessing the Temporal Relationship Between Cognition and Gait: Slow Gait Predicts Cognitive Decline in the Mayo Clinic Study of Aging. J Gerontol Ser A Biol Sci Med Sci. 2013;68(8):929-37.

9. Cohen JA, Verghese J. Gait and dementia. In: Handbook of clinical neurology [Internet]. Vol. 167. [Sem local]: Elsevier; 2019 [acesso em 09 set. 2019]. Chapter 22. p. 419-27. Disponível em: http://dx.doi. org/10.1016/B978-0-12-804766-8.00022-4

10. Verghese J, Wang C, Lipton RB, Holtzer R. Motoric cognitive risk syndrome and the risk of dementia. J Gerontol Ser A Biol Sci Med Sci. 2013;68(4):412-8.

11. Verghese J, Annweiler C, Ayers E, Bennett DA, Stephanie A, Buchman AS, et al. Motoric cognitive risk syndrome: multicountry prevalence and dementia risk. Neurology. 2014;83(8):718-26.

12. Allali G, Ayers EI, Verghese J. Motoric Cognitive Risk Syndrome Subtypes and Cognitive Profiles. J Gerontol Ser A Biol Sci Med Sci. 2016;71(3):378-84.

13. Doi T, Verghese J, Shimada H, Makizako $\mathrm{H}$, Tsutsumimoto K, Hotta R, et al. Motoric Cognitive Risk Syndrome: prevalence and Risk Factors in Japanese Seniors. J Am Med Dir Assoc. 2015;16(12):21-5. Disponível em: http://dx.doi. org/10.1016/j.jamda.2015.09.003

14. Maguire FJ, Killane I, Creagh AP, Donoghue O, Kenny RA, Reilly RB. Baseline Association of Motoric Cognitive Risk Syndrome With Sustained Attention, Memory, and Global Cognition. J Am Med Dir Assoc. 2018;19(1):53-8. Disponível em: https:// doi.org/10.1016/j.jamda.2017.07.016 
15. Callisaya ML, Ayers E, Barzilai N, Ferrucci L, Guralnik JM, Lipton RB, et al. Motoric Cognitive Risk Syndrome and Falls Risk: A Multi-Center Study. J Alzheimers Dis. 2016;53(3):1043-52.

16. Chhetri JK, Chan P, Vellas B, Cesari M. Motoric cognitive risk syndrome: Predictor of dementia and age-related negative outcomes. Front Med. 2017;4:1-8.

17. Doi T, Shimada H, Makizako H, Tsutsumimoto K, Verghese J, Suzuki T. Motoric Cognitive Risk Syndrome: association with Incident Dementia and Disability. J Alzheimer's Dis. 2017;59(1):77-84

18. Nichols E, Szoeke CEI, Vollset SE, Abbasi N, Abd-Allah F, Abdela J, et al. Global, regional, and national burden of Alzheimer's disease and other dementias, 1990-2016: a systematic analysis for the Global Burden of Disease Study 2016. Lancet Neurol. 2019;18(1):88-106.

19. Saraiva LB, Oliveira FA, de Almeida ANS, de Jesus Moreira DJ, Barbosa RGB. Avaliação geriátrica ampla e sua utilização no cuidado de enfermagem a pessoas idosas. J Health Sci. 2017;19(4):262-7.

20. Brucki SMD, Nitrin R, Caramelli P, Bertolucci PHF, Okamoto IH. Sugestões para o uso do mini-exame do estado mental no Brasil. Arq Neuropsiquiatr. 2003;61(3 B):777-81.

21. Katz S, Ford AB, Moskowitz RW, Jackson BA, Jaffe MW. Studies of Illness in the Aged. The Index of ADL: a Standardized Measure of Biological and Psychosocial Function. JAMA. 1963;185(12):914-9.

22. Lawton M, Brody E. Assessment of older people: self maintaining and instrumental activities of daily living. Gerontologist. 1969;9:1979-86.

23. Lino VTS, Pereira SRM, Camacho LAB, Ribeiro Filho ST, Buksman S. Adaptação transcultural da Escala de Independência em Atividades da Vida Diária (Escala de Katz). Cad Saúde Pública. 2008;24(1):103-12.

24. Nakano MM. Versão Brasileira da Short Physical Performance Battery - SPPB: Adaptação cultural e estudo da confiabilidade [Dissertação]. Campinas: Universidade Estadual de Campinas, Faculdade de Educação; 2007 [acesso em 09 set. 2019]. Disponível em: http://repositorio.unicamp.br/handle/ REPOSIP/252485

25. Torres GV, dod Reis LA, dos Reis LA. Assessment of functional capacity in elderly residents of an outlying area in the hinterland of Bahia/Northeast Brazil. Arq Neuropsiquiatr. 2010;68(1):39-43.
26. Patrizio E, Calvani R, Marzetti E, Cesari M. Physical Functional Assessment in Older Adults. J Frailty Aging [Internet]. 2021;10(2):141-9. Disponível em: http://dx.doi.org/10.14283/jfa.2020.61

27. Semba RD, Tian Q, Carlson MC, Xue QL, Ferrucci L. Motoric cognitive risk syndrome: Integration of two early harbingers of dementia in older adults. Ageing Res Rev. 2020;58:e101022. Disponível em: https://doi.org/10.1016/j.arr.2020.101022

28. Shim H, Kim M, Won CW. Motoric cognitive risk syndrome using three-item recall test and its associations with fall-related outcomes: the korean frailty and aging cohort study. Int J Environ Res Public Health. 2020;17(10):1-16.

29. Zhang L, Feng BL, Wang CY, Zhang Y, Lin P, Zhang YL, et al. Prevalence and factors associated with motoric cognitive risk syndrome in communitydwelling older Chinese: a cross-sectional study. Eur J Neurol. 2020;27(7):1137-45.

30. Guimarães HPN, Simões MC, Pardi GR. Perfil sociodemográfico, condições de saúde e hábitos alimentares de idosos acompanhados em ambulatório geriátrico. Rev Fam Ciclos Vida Saúde Contexto Soc. 2019;7(2):186-99.

31. Ayers E, Verghese J. Gait Dysfunction in Motoric Cognitive Risk Syndrome. J Alzheimer's Dis. 2019;71(Suppl1):95-103.

32. George CJ, Verghese J. Motoric Cognitive Risk Syndrome in Polypharmacy. J Am Geriatr Soc. 2020;68(5):1072-7.

33. Doi T, Verghese J, Shimada H. Motoric Cognitive Risk Syndrome : Prevalence and Risk Factors in Japanese Seniors. J Am Med Dir Assoc. 2015;16(12):21-5.

34. Beauchet O, Sekhon H, Launay CP, Gaudreau P, Morais JA, Allali G. Relationship between motoric cognitive risk syndrome, cardiovascular risk factors and diseases, and incident cognitive impairment: results from the "NuAge" study. Maturitas. 2020;138:51-7. Disponível em: https://doi. org/10.1016/j.maturitas.2020.05.007

35. Lauretani F, Meschi T, Ticinesi A, Maggio M. "Brainmuscle loop" in the fragility of older persons : from pathophysiology to new organizing models. Aging Clin Exp Res. 2017;29(6):1305-11. 